\title{
Patching DFT, T-duality and gerbes
}

\section{P.S. Howe and G. Papadopoulos}

Department of Mathematics, King's College London, Strand, London WC2R 2LS, U.K.

E-mail: paul.howe@kcl.ac.uk, george.papadopoulos@kcl.ac.uk

ABSTRACT: We clarify the role of the dual coordinates as described from the perspectives of the Buscher T-duality rules and Double Field Theory. We show that the T-duality angular dual coordinates cannot be identified with Double Field Theory dual coordinates in any of the proposals that have been made in the literature for patching the doubled spaces. In particular, we show with explicit examples that the T-duality angular dual coordinates can have non-trivial transition functions over a spacetime and that their identification with the Double Field Theory dual coordinates is in conflict with proposals in which the latter remain inert under the patching of the B-field. We then demonstrate that the Double Field Theory coordinates can be identified with some C-space coordinates and that the T-dual spaces of a spacetime are subspaces of the gerbe in C-space. The construction provides a description of both the local $O(d, d)$ symmetry and the T-dual spaces of spacetime.

Keywords: String Duality, Differential and Algebraic Geometry, Superstrings and Heterotic Strings

ARXIV EPRINT: 1612.07968 


\section{Contents}

1 Introduction 1

2 T-duality rules and patching $\quad 4$

2.1 T-duality rules 4

2.2 The T-dual circle topologically twists over the spacetime 6

$\begin{array}{ll}2.3 \text { A patching approach to T-duality } & 7\end{array}$

2.4 T-duality on $T^{3}$ with flux 8

3 Double field theory finite transformations $\quad 8$

$\begin{array}{lll}4 & \text { Patching } & \mathbf{1 0}\end{array}$

$\begin{array}{lll}4.1 & B \text {-dependent patching for dual coordinates } & 11\end{array}$

$\begin{array}{lll}4.2 & B \text {-independent patching for dual coordinates } & 12\end{array}$

5 A new proposal $\quad 13$

$\begin{array}{lll}5.1 & \text { C-spaces and DFT coordinates } & 13\end{array}$

$\begin{array}{lll}5.2 & \text { Gerbes and Buscher rules } & 14\end{array}$

$\begin{array}{lll}5.3 & \text { Summary of the proposal } & 15\end{array}$

6 Conclusions $\quad 16$

\section{Introduction}

One of the requirements for the consistent formulation of double and exceptional field theories is a description of the patching conditions of doubled and exceptional spaces that underpin these theories. Let us for simplicity focus on doubled spaces as many more results for these are known. Doubled spaces arise by adding to the spacetime coordinates $x$ a set of dual coordinates $\tilde{x}$. In double field theory (DFT), the new coordinates are as many as those of the spacetime.

The question that arises is how these new coordinates patch. There are two main approaches in the literature for this. In the first approach, it is proposed that the dual coordinates $\tilde{x}$ patching transformations depend on the transition functions of the $B$-field. There are various suggestions for such dependence. Two such suggestions can be found in $[1,2]$ and [3].

Another approach, advocated in [4], asserts that the patching conditions of the dual coordinates $\tilde{x}$ can be arranged such that they do not depend on the transition functions of the $B$-field. In such a case, the doubled space of any string background spacetime $M$ is either a product space $M \times Q$, where $Q$ can be chosen as $\mathbb{R}^{n}$ or $T^{n}$, or the cotangent 
bundle $T^{*} M$. The dual coordinates in this case become forgetful, in the sense that they are inert under $B$-field gauge transformations.

In addition, a recent proposal for DFT for some coset spaces was made in [5], following on from an analysis of DFT for Wess-Zumino-Witten models presented in $[6,7]$.

One of the difficulties in deciding the way that the dual coordinates should patch is the uncertainty of which criteria one should apply. A selection of such criteria is as follows:

- The doubled spaces patch in such a way that is consistent with the dual spaces obtained via the Buscher T-duality rules.

- The patching of double spaces is such that it requires for consistency the Dirac quantisation property of the 3 -form flux.

- The doubled spaces satisfy the topological geometrisation condition.

- Doubled spaces can be constructed for all backgrounds with 3-form flux.

- Generalised geometry emerges naturally on doubled spaces.

The first criterion is perhaps the most conservative one. Whatever the patching of doubled spaces is, it should reproduce both locally and globally the results that arise after applying the Buscher T-duality rules. After all these produce the only explicit examples we know. Locally this is indeed the case through the use of $O(d, d)$ duality transformations [811 ] on the fields. However, we shall see that globally the patching conditions of the doubled spaces do not reproduce the results obtained from Buscher rules.

Moreover, it is worth mentioning that DFT has raised the expectations of what can be described. As the transformations of DFT make no mention of isometries that are instrumental in the Buscher rules, there is some expectation that the doubled spaces can be used to describe a dual space which arises after dualising all spacetime directions. Another aspect of the dualisation of the whole spacetime is the idea of geometrisation, i.e. the notion that the theory which includes the spacetime metric and the 3-form field strength can be described in terms of metric data only. This is analogous to Kaluza-Klein theory which provides a geometrisation for a 2-form field strength.

The second criterion is an extrapolation of a similar result that arises in Kaluza-Klein theory. The construction of the Kaluza-Klein space is achieved after restricting the 2-form field strength to represent the first Chern class of a line bundle. In turn the flux of the 2 -form is required to obey the Dirac quantisation condition.

The third criterion is also posed in analogy with the Kaluza-Klein theory. It states that the pull-back of the 3-form field strength on whatever a consistent description of doubled space is, or a generalisation of it, must represent the trivial cohomology class [13]. This has several consequences such as, for example, that the dual coordinates must have a non-trivial topology and non-trivial transition functions over the spacetime.

The fourth criterion is a natural one from the point of view of DFT. In all proposals made in the literature for the theory, there is no restriction mentioned on the backgrounds.

The fifth criterion is introduced because in generalised geometry the T-duality group $O(d, d)$ arises naturally as the (sub)group of automorphisms of a vector bundle. So the 
expectation is that in a consistent formulation of the doubled space this bundle should arise naturally. In fact it is expected to be related to, if not identified with, its tangent bundle.

There are several proposals in the literature on how the doubled spaces might patch and some analysis of how they measure against the criteria mentioned above. In particular, the patching of doubled spaces under the transformations proposed in $[1,2]$ has been investigated in [12] where it was shown that consistency on 4-overlaps requires that the 3 -form field strength $H$ must be exact. To resolve the patching issue, $\mathrm{C}$-spaces, essentially local descriptions of gerbes, have been proposed in [13]. They exhibit consistent patching with a cohomologically non-trivial $H$ and locally contain the doubled spaces, but generically they have more coordinates than doubled spaces. Indeed, in the case of non-trivial $H$-fields they do not have well-defined global dimensionalities.

More recently, two new proposals for patching doubled spaces have been put forward [3, 4]. In this paper, we shall consider these two proposals and investigate them in the light of the criteria mentioned above. First we shall clarify some aspects of the patching conditions proposed in [3] and demonstrate that, up to an allowed redefinition of the dual coordinates and choice of transition functions for $B$ at double overlaps, the patching conditions of the dual coordinates do not depend on the transition functions of the $B$-field. As a result, for these choices, the dual coordinates of the doubled space remain inert under patching which in turn implies that this proposal is related to that of [4].

The proposal made in [4] states that the dual coordinates of a doubled space remain inert under patching and the transformations induced by the form part of a generalised vector acting infinitesimally with a generalised Lie derivative on the fields are not coordinate transformations but rather gauge transformations of the $B$-field. As a result the dual coordinates can be forgetful and the spacetime geometry is described by a generalised geometry structure and a splitting of the generalised geometry bundle induced by the $B$ field interpreted as a gerbe connection.

In the proposal of [5] for DFT on group manifolds, the doubled space is a group manifold with the physical space embedded into it as a Lagrangian type of submanifold, after the strong section condition is imposed, while the T-dual space corresponds to a different embedding. In this case both the physical and dual coordinates are non-trivially patched.

In what follows we give a detailed analysis of the T-duality pair of $S^{3}$ with $N$ units of $H$-charge and the lens space $L_{N}^{3}=S^{3} / \mathbb{Z}_{N}$ with 1 unit of $H$ charge. We show that the dual circle twists topologically non-trivially over the spacetime $L_{N}^{3}$ and therefore that either DFT dual coordinates cannot be identified ${ }^{1}$ with the T-duality angular coordinates, or that the doubled spaces patching proposed in [3] and [4] is not consistent globally with the T-duality rules. We also generalise this to other T-dual pairs including an example of T-dual spaces constructed from the 3-torus with H-flux background.

Note that a conflict between T-duality and the strong section condition in doubled spaces had been pointed out before from a different perspective in $[14,15]$. There a resolution was proposed by allowing additional transformations which preserve the split signature metric on the doubled space but do not satisfy the strong section condition.

\footnotetext{
${ }^{1}$ As our results are topological, this rules out all continuous and even homotopic identifications.
} 
We then go on to propose a scenario based on C-spaces and the Hitchin-Chatterjee definition of a gerbe in which both the local $O(d, d)$ symmetry and the Buscher T-dual spaces can be consistently described. We propose an identification of the DFT coordinate $\tilde{x}$ of [4], which transforms as a 1-form, with a coordinate that arises in the C-space construction [13]. We then demonstrate how the T-dual space $\tilde{M}$ of a spacetime $M$ with $H$-flux and which is a circle fibration can be identified as a subspace of the total space of the gerbe associated to $H$ on $M$. We also provide explicit examples of this which include the description of the T-dual lens space $L_{N}^{3}=S^{3} / \mathbb{Z}_{N}$ of $S^{3}$ with $N$ units of $H$-charge as a subspace of the total space of a gerbe on $S^{3}$. The latter can be described as the union of $S^{3}$ with a circle bundle with first Chern class $N$ over an open neighbourhood of the equatorial $S^{2}$ of $S^{3}$. The $L_{N}^{3}$ subspace of the gerbe is the restriction of this circle bundle over the equatorial $S^{2}$ of $S^{3}$. We also give a similar construction for a T-dual space associated 3torus background with H-flux. As the angular coordinates that arise naturally in the gerbe construction, and which are required for the identification of the T-dual spaces of spacetime as subspaces of gerbes, are not included in doubled spaces and therefore not in DFT, we conclude that, for the consistent description of a theory with manifest Buscher T-duality symmetry, additional coordinates are required in addition to those of doubled spaces.

The paper is organised as follows: in section 2, we give the necessary and sufficient conditions for the T-dual circle to (topologically) twist over a spacetime in a manner consistent with the Buscher rules. We also prove that the dual circle of the lens space $L_{N}^{3}$, viewed as a circle fibration over $S^{2}$, and that of $T^{3}$ with $H$-flux, topologically twist over the spacetime. In section 3, we review the proposals for patching DFT that have appeared in the literature and in section 4 we investigate them from a patching point of view concluding that they do not describe the topological twist of the dual circles. In section 5 , we explore the relation between doubled spaces and C-spaces, explain how local $O(d, d)$ symmetry arises, and present a gerbe construction for all spacetimes which are circle fibrations and have some $H$-flux which allows for the identification of the T-dual space as a subspace of the gerbe. We also present explicit examples based on $S^{3}$ and $T^{3}$ with H-flux backgrounds. In section 6, we present our conclusions.

\section{T-duality rules and patching}

\section{$2.1 \quad$ T-duality rules}

To describe the Buscher T-duality rules one assumes that the spacetime $M$ admits an $S^{1}$ group action generated by a vector field $X$ which leaves the common sector fields, the metric $g$, 3-form field strength $H$ and dilaton $\Phi$, invariant. Adapting coordinates along $X=\frac{\partial}{\partial \theta}$, the metric and 2-form gauge potential can be written as

$$
d s^{2}=V^{2}\left(d \theta+q_{i} d x^{i}\right)^{2}+g_{i j} d x^{i} d x^{j}, \quad B=\left(d \theta+q_{i} d x^{i}\right) \wedge p_{j} d x^{j}+\frac{1}{2} b_{i j} d x^{i} \wedge d x^{j}
$$


After performing a T-duality transformation, the dual metric, 2-form gauge potential and dilaton read

$$
\begin{aligned}
& d \tilde{s}^{2}=V^{-2}\left(d \tilde{\theta}+p_{i} d x^{i}\right)^{2}+g_{i j} d x^{i} d x^{j}, \quad \tilde{B}=\left(d \tilde{\theta}+p_{i} d x^{i}\right) \wedge q_{j} d x^{j}+\frac{1}{2} b_{i j} d x^{i} \wedge d x^{j}, \\
& e^{2 \tilde{\Phi}}=e^{2 \Phi} V^{-2}
\end{aligned}
$$

where a new angular coordinate $\tilde{\theta}$ has now been introduced. This is referred as the T-dual coordinate of $\theta$ and the associated circle as the dual circle, which we denote $\tilde{S}^{1}$. The coordinates of $\left(x^{i}, \tilde{\theta}\right)$ are those of a new spacetime $\tilde{M}$ which, apart from having different geometry, can also have different topology to that of $M$. Furthermore $\tilde{M}$ again admits a $\tilde{S}^{1}$ action given by translations in $\tilde{\theta}$. Another significant issue, which will be of central focus in what follows, is that the T-dual coordinate $\tilde{\theta}$ can have non-trivial patching conditions over the original spacetime $M$ (or vice versa). These are given by some of the transition functions of the $B$-field. As can be seen from (2.1), $p_{i}$ will transform under a $B$-field transformation and this will induce a transformation of $\tilde{\theta}$ in (2.2) in order for the T-dual metric to remain invariant.

The original spacetime $M$ together with its dual $\tilde{M}$ can be put together to construct an enhanced space. To see this observe that the space of orbits of the $S^{1}$ action on $M$ and of the $\tilde{S}^{1}$ action on $\tilde{M}$ are the same, $M / S^{1}=\tilde{M} / \tilde{S}^{1}=Q$. To avoid complications with fixed points, let us assume from now on that the action of $S^{1}$ on both spaces is free. ${ }^{2}$ In such a case, one can construct a torus bundle $P\left(Q, T^{2}\right)$ over $Q$. The torus bundles are classified by elements in $H^{2}(Q, \mathbb{Z}) \oplus H^{2}(Q, \mathbb{Z})$ which are the first Chern classes of $M$ and $\tilde{M}$ viewed as circle bundles over $Q$. In [17], $P\left(Q, T^{2}\right)$ is referred to as the correspondence space. In particular, the first Chern classes are represented by the 2 -forms $\frac{1}{2 \pi} d q$ and $\frac{1}{2 \pi} d p$, respectively, with $p=p_{i} d x^{i}$ and similarly for $q$.

We therefore have the diagram

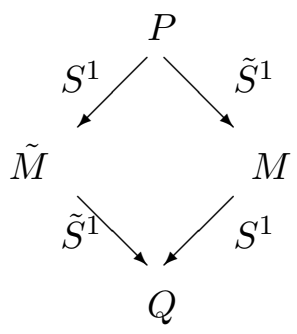

We can also define two-forms $F$ and $\tilde{F}$ on $Q$ by integrating $\tilde{H}$ over $\tilde{S}^{1}$ and $H$ over $S^{1}$ respectively. Here, from the T-duality rules,

$$
\begin{aligned}
& H=d B=-d \theta \wedge d p+h+d(q \wedge p) \\
& \tilde{H}=d \tilde{B}=-d \tilde{\theta} \wedge d q+h+d(p \wedge q),
\end{aligned}
$$

\footnotetext{
${ }^{2}$ Otherwise, one can use the slice theorem to remove the fixed points and repeat the same analysis on the remaining space.
} 
where $h=d b$. So

$$
F=-\frac{1}{4 \pi^{2}} \int_{\tilde{S}^{1}} \tilde{H} ; \quad \tilde{F}=-\frac{1}{4 \pi^{2}} \int_{S^{1}} H .
$$

Equations (2.4) and (2.5), together with the fact that both $M$ and $\tilde{M}$ have the same quotient $Q$ as circle bundles, were specified as the required conditions for the two spaces to be T-dual in [18].

\subsection{The T-dual circle topologically twists over the spacetime}

Although the T-dual coordinates $\tilde{\theta}$ have non-trivial transition functions over $Q$, it does not necessarily mean that they are (topologically) twisted over the spacetime $M$. To settle this question, let us examine an example in detail. This is the well-known T-dual pair of $S^{3}$ with $\mathrm{N}$-units of $H$ flux and the 3 -dimensional lens space $L_{N}^{3}$ with 1-unit of $H$ charge. It is useful to note that $L_{N}^{3}$ is the space of orbits of $\mathbb{Z}_{N}$ on $S^{3}$ where the generator $g=\exp 2 \pi i / N$ of $\mathbb{Z}_{N}$ acts as $v_{r} \rightarrow g v_{r}$, where $v_{r}$ are complex numbers such that $v_{1} \bar{v}_{1}+v_{2} \bar{v}_{2}=1$.

Both spaces $S^{3}$ and $L_{N}^{3}$ are circle fibrations over $S^{2}, Q=S^{2}$. Moreover the first Chern class of these fibrations is $c_{1}\left(S^{3}\right)=u$ and $c_{1}\left(L_{N}^{3}\right)=N u$, respectively, where $u$ is the generator of $H^{2}\left(S^{2}, \mathbb{Z}\right)$. Furthermore the cohomology groups of $S^{3}$ and $L_{N}^{3}$ are

$$
\begin{aligned}
H^{0}\left(S^{3}, \mathbb{Z}\right) & =H^{3}\left(S^{3}, \mathbb{Z}\right)=\mathbb{Z}, & H^{1}\left(S^{3}, \mathbb{Z}\right) & =H^{2}\left(S^{3}, \mathbb{Z}\right)=0, \\
H^{0}\left(L_{N}^{3}, \mathbb{Z}\right) & =H^{3}\left(L_{N}^{3}, \mathbb{Z}\right)=\mathbb{Z}, & H^{1}\left(L_{N}^{3}, \mathbb{Z}\right) & =0, \quad H^{2}\left(L_{N}^{3}, \mathbb{Z}\right)=\mathbb{Z}_{N} .
\end{aligned}
$$

Next consider the $T^{2}$ fibration $P=P\left(T^{2}, S^{2}\right)$ with first Chern classes $c_{1}(P)=u$ and $c_{1}(P)=N u$. In fact $P=\left(S^{1} \times S^{3}\right) / \mathbb{Z}_{N}$, where now the generator $g$ of $\mathbb{Z}_{N}$ acts as $\left(a, v_{r}\right) \rightarrow\left(g a, g v_{r}\right)$ and $|a|=1, a \in \mathbb{C}$. It turns out that the cohomology of $P$ can be computed and can be found that

$$
H^{0}(P, \mathbb{Z})=H^{1}(P, \mathbb{Z})=H^{3}\left(S^{3}, \mathbb{Z}\right)=H^{4}(P, \mathbb{Z})=\mathbb{Z}, \quad H^{2}(P, \mathbb{Z})=0 .
$$

In particular observe that the middle cohomology of $P$ vanishes.

To continue observe that $P$ can be viewed as a circle fibration over either $S^{3}$ or $L_{N}^{3}$. Consider first $P$ as a circle fibration over $S^{3}$. This fibration is obtained after considering the group action $\left[a, v_{r}\right] \rightarrow\left[a z, z v_{r}\right]$, where $z \in S^{1} \subset \mathbb{C},|z|=1$ is the group element and $\left[a, v_{r}\right]$ denotes the orbit of $Z_{N}$ represented by $\left(a, v_{r}\right)$. In fact notice that $S^{1} / \mathbb{Z}_{N}=S^{1}$ acts freely. As $H^{2}\left(S^{3}, \mathbb{Z}\right)=0$, all circle bundles over $S^{3}$ are topologically trivial. As a result $P$ is a topological product $S^{1} \times S^{3}$. One therefore concludes that the dual coordinate $\tilde{\theta}$ does not twist over the spacetime $S^{3}$.

However the T-dual Lens space $L_{N}^{3}$ can also be considered as the spacetime, and so $S^{3}$ can be thought as its T-dual. Note that $H^{2}\left(L_{N}^{3}, \mathbb{Z}\right)=\mathbb{Z}_{N}$ and so $L_{N}^{3}$ admits topologically non-trivial circle bundles. The fibration of $P$ over $L_{N}^{3}$ is constructed by considering the circle action $\left[a, v_{r}\right] \rightarrow\left[a z, v_{r}\right]$. If $P$ was a trivial topological product $S^{1} \times L_{N}^{3}$, the Künneth formula for computing the cohomology of the topological product of two spaces would have implied that

$$
H^{2}(P, \mathbb{Z})=H^{2}\left(L_{N}^{3}, H^{0}\left(S^{1}, \mathbb{Z}\right)\right)=H^{2}\left(L_{N}^{3}, \mathbb{Z}\right)=\mathbb{Z}_{N}
$$


This is a contradiction as the second cohomology of $P$ vanishes (2.7). Therefore $P$ is a topologically twisted product of $S^{1}$ and $L_{N}^{3}$. As a result, the dual $\theta$ coordinate has non-trivial patching conditions over the spacetime $L_{N}^{3}$.

Incidentally, observe that $P$ satisfies a partial version of the topological geometrisation condition of [13]. Both the $S^{3}$ backgrounds and its dual $L_{N}^{3}$ have non-trivial $H$ fluxes. As a result, the T-duality operation does not geometrise all of the B-flux, so that one does not expect that the pull back of $H$ or $\tilde{H}$ on $P$ will represent the trivial class in $H^{3}(P, \mathbb{Z})$. Instead the topological geometrisation condition manifests itself as follows: pulling back $H$ and $\tilde{H}$ onto $P$, one may have expected that these represent two independent cohomology classes in $H^{3}(P, \mathbb{Z})$, but this is not the case. $H^{3}(P, \mathbb{Z})$ has one generator and the linear combination $N H-\tilde{H}$ represents the trivial class in $H^{3}(P, \mathbb{Z})$, where we have suppressed the pull-back operations. This is because part of the information of the transitions functions of $H$ and $\tilde{H}$ is stored in the patching conditions of $P$.

The example we have given above can be generalised to include $T^{n}$ actions and thus T-duality in more than one direction. However, for the purpose of this paper, the example we have investigated will suffice.

To conclude, the Buscher T-duality rules allow for the possibility that the dual circle has a non-trivial topological twist over the spacetime, so that the dual angular coordinates can have non-trivial patching conditions over the spacetime. As we have seen, this situation does indeed arise in explicit examples.

\subsection{A patching approach to T-duality}

To give a bit more insight into the construction of circle bundle over a space and its relation to the T-dual pairs, let us first describe how the third cohomology group of the spacetime is constructed from the cohomology of $S^{1}$ and that of $Q \cdot{ }^{3}$ Assuming again that $S^{1}$ acts freely on the spacetime $M$ and that $Q$ is simply connected, one can use the method of spectral sequences to determine $H^{3}(M, \mathbb{Z})$ from $H^{1}\left(S^{1}, \mathbb{Z}\right), H^{2}(Q, \mathbb{Z})$ and $H^{3}(Q, \mathbb{Z})$. The construction is rather intuitive. The elements of $H^{3}(M, \mathbb{Z})$ either are generated by au, where $a$ is the generator of $H^{1}\left(S^{1}, \mathbb{Z}\right)$ and $u$ are generators of $H^{2}(Q)$, or they are pulledback from elements in $H^{3}(Q, \mathbb{Z})$ with the projection map. This is precisely the case if $H^{4}(Q, \mathbb{Z})=0$. If on the other hand $H^{4}(Q, \mathbb{Z}) \neq 0$, then only some of classes generated by au may represent elements in $H^{3}(M, \mathbb{Z})$. In either case, the 3 -form field strength $H$ in cohomology can be written as $[H]=a w+v$, where $w \in H^{2}(Q, \mathbb{Z})$ and $v \in H^{3}(Q, \mathbb{Z})$, and where the pull-back operation on $v$ has been suppressed.

It is clear from the T-duality rules stated in (2.1) and (2.2) that the component of $H$ that take an active part in the T-duality transformations is represented by aw. Assuming that $w \in H^{2}(Q, \mathbb{Z})$, the dual space $\tilde{M}$ as a circle bundle has first Chern class $w$. For later applications, let us assume that $w$ is represented by a 2 -form $\tilde{F}^{2}$. The construction of $\tilde{M}$ can be made using a good cover $\left\{U_{\alpha}\right\}_{\alpha \in I}$ on $Q$. Then, using the Poincaré lemma on $U_{\alpha}$, and on double and triple overlaps, $U_{\alpha \beta}=U_{\alpha} \cap U_{\beta}$ and $U_{\alpha \beta \gamma}=U_{\alpha} \cap U_{\beta} \cap U_{\gamma}$ respectively,

\footnotetext{
${ }^{3}$ In this subsection we allow $Q$ to have more than two dimensions in the general discussion.
} 
we find

$$
\tilde{F}_{\alpha}^{2}=d C_{\alpha}^{1}, \quad-C_{\alpha}^{1}+C_{\beta}^{1}=d a_{\alpha \beta}^{0}, \quad a_{\beta \gamma}^{0}-a_{\alpha \gamma}^{0}+a_{\alpha \beta}^{0}=n_{\alpha \beta \gamma},
$$

where $C$ is the 1 -form gauge potential, $a^{0}$ are the transition functions on double overlaps and $n$ are constants. The latter lie in $2 \pi \mathbb{Z}$ as $\frac{1}{2 \pi} \omega^{2}$ represents a class in $H^{2}(Q, \mathbb{Z})$. Then $\tilde{M}$ is constructed by introducing an angular coordinate $\tilde{\theta}$ and after imposing the patching conditions

$$
\tilde{\theta}_{\alpha}-\tilde{\theta}_{\beta}-a_{\alpha \beta}^{0}=0 \bmod 2 \pi \mathbb{Z} .
$$

These patching conditions are consistent on triple overlaps as $n_{\alpha \beta \gamma} \in 2 \pi \mathbb{Z}$.

Making use of the above, we can state the criterion for whether the dual angular coordinate has non-trivial transition functions over the spacetime. Indeed, writing $[H]=$ $a w+v$ and $[\tilde{H}]=a \tilde{w}+\tilde{v}$, we observe that the dual angular coordinate $\tilde{\theta}$ has non-trivial transition functions over the spacetime iff $w$ represents a non-trivial class in $H^{2}(M, \mathbb{Z})$, where the pull-back operation from $H^{*}(Q, \mathbb{Z})$ to $H^{*}(M, \mathbb{Z})$ has been suppressed. Similarly, the angular coordinate $\theta$ has non-trivial transition functions over the dual space $\tilde{M}$ iff $\tilde{w}$ represents a non-trivial class in $H^{2}(\tilde{M}, \mathbb{Z})$. The classes $w$ and $\tilde{w}$ are represented by the forms $\tilde{F}$ and $F$ in (2.5) respectively.

\section{$2.4 \quad$ T-duality on $T^{3}$ with flux}

We can use the results of the previous section to demonstrate that the T-dual angular coordinate of $T^{3}$ with flux also is twisted over the spacetime. For this denote the angular coordinates of $T^{3}$ with $\left(\psi_{1}, \psi_{2}, \psi_{3}\right), 0 \leq \psi_{i}<2 \pi, i=1,2,3$. The metric and flux are given as

$$
d s^{2}=\left(d \psi_{1}\right)^{2}+\left(d \psi_{2}\right)^{2}+\left(d \psi_{3}\right)^{2}, \quad H=-\frac{N}{4 \pi^{2}} d \psi_{1} \wedge d \psi_{2} \wedge d \psi_{3},
$$

where $N \in \mathbb{Z}$. If we choose as a T-duality direction $\psi_{1}$ and solve for the gauge potential as $B=\frac{N}{4 \pi^{2}} \psi_{2} d \psi_{1} \wedge d \psi_{3}$, then

$$
p=\frac{N}{2 \pi} \psi_{2} d \psi_{3},
$$

where the Killing vector field along the T-duality has been normalised as $2 \pi \partial_{\psi_{1}}$. As it has been explained in the previous section, the dual coordinate topologically twists over the spacetime iff the pull-back of $d p$ represents a non-trivial cohomology class. Indeed

$$
d p=\frac{N}{2 \pi} d \psi_{2} \wedge d \psi_{3}
$$

and its pull-back on $T^{3}$ is a non-trivial class as $\frac{1}{2 \pi} d \psi_{2} \wedge d \psi_{3}$ represents one of the three generators of $H^{2}\left(T^{3}, \mathbb{Z}\right)$.

\section{Double field theory finite transformations}

There has been extensive work in the literature to determine the allowed finite transformations of DFT. A concise description of all possibilities and the sources can be found in [4]. Here after imposing the strong section condition, we shall briefly summarise the 
finite transformations proposed as well as their induced action on the $B$-field. This will suffice for the purpose of the analysis that follows below.

First let us begin with the proposal of $[1,2]$. In this proposal, the doubled space coordinates $\left(x^{i}, \tilde{x}_{i}\right)$ transform as

$$
x^{i}=x^{\prime i}\left(x^{j}\right), \quad \tilde{x}_{i}^{\prime}=\tilde{x}_{i}-v_{i}(x),
$$

and the induced transformation on the $B$ field is

$$
B_{i j}^{\prime}\left(x^{\prime}\right)=\frac{\partial x^{k}}{\partial x^{\prime i}} \frac{\partial x^{l}}{\partial x^{\prime j}}\left(B_{k l}(x)+\frac{1}{2}\left(\frac{\partial v_{l}}{\partial x^{k}}-\frac{\partial v_{k}}{\partial x^{l}}\right)\right)+\frac{1}{2}\left(\frac{\partial x^{k}}{\partial x^{\prime i}} \frac{\partial v_{j}}{\partial x^{k}}-\frac{\partial x^{k}}{\partial x^{\prime j}} \frac{\partial v_{i}}{\partial x^{k}}\right) .
$$

Observe that the spacetime coordinates transform with the usual diffeomorphisms while the dual coordinates transform with a shift whose parameter depends only on the spacetime coordinates. A modification of this proposal in the context of DFT was suggested in [16]; however, the transformations given in [16] reduce to the above after the strong section condition has been imposed.

Another proposal for the finite transformation of DFT was put forward in [3]. For this, a closed 2-form was introduced $b, d b=0$ which transforms as

$$
b_{i j}^{\prime}\left(x^{\prime}\right)=\frac{\partial x^{k}}{\partial x^{\prime i}} \frac{\partial x^{l}}{\partial x^{\prime j}}\left(\left(b_{k l}+\partial_{k} v_{l}-\partial_{l} v_{k}\right)(x)\right),
$$

while $\mathbf{B}:=B-b$ is taken to transform tensorially:

$$
\mathbf{B}_{i j}^{\prime}\left(x^{\prime}\right)=\frac{\partial x^{k}}{\partial x^{\prime i}} \frac{\partial x^{l}}{\partial x^{\prime j}} \mathbf{B}_{k l}(x) .
$$

This implies that the $B$-field transforms as

$$
\left.B_{i j}^{\prime}\left(x^{\prime}\right)=\frac{\partial x^{k}}{\partial x^{\prime i}} \frac{\partial x^{l}}{\partial x^{\prime j}}\left(B_{k l}+\partial_{k} v_{l}-\partial_{l} v_{k}\right)(x)\right),
$$

i.e. in the same way as $b$. In these equations $v$ depends only on the spacetime coordinates. The doubled space coordinate transformations are taken to be

$$
x^{i}=x^{\prime i}\left(x^{j}\right), \quad \tilde{x}_{i}^{\prime}=\tilde{x}_{i}+v_{i}(x) .
$$

More recently a new proposal has been put forward [4]. The doubled space coordinates transform as

$$
x^{\prime i}=x^{\prime i}\left(x^{j}\right), \quad \tilde{x}_{i}^{\prime}=\tilde{x}_{i},
$$

i.e. the spacetime coordinates transform with diffeomorphisms while the dual coordinates remain inert with respect to $B$-field gauge transformations.

The $B$-field transforms as

$$
B_{i j}^{\prime}\left(x^{\prime}\right)=\frac{\partial x^{k}}{\partial x^{\prime i}} \frac{\partial x^{l}}{\partial x^{\prime j}}\left(B_{k l}(x)+\left(\partial_{k} v_{l}-\partial_{l} v_{k}\right)(x)\right),
$$

i.e. in the same way as in [3]. 
The reason that the dual coordinates $\tilde{x}$ do not transform under the $B$-field gauge transformations is because the component $\tilde{v}_{j}$ of the generalised infinitesimal vector,

$$
V^{M}=\left(\begin{array}{c}
v^{i} \\
\tilde{v}_{j}
\end{array}\right)
$$

that enters in the generalised Lie derivative acting on the fields, is identified as the parameter of an infinitesimal gauge transformation of the $B$ field viewed as a gerbe connection. In other words $\tilde{v}_{j}$ is viewed as (the parameter of) a gauge transformation rather than as a coordinate transformation. Moreover the gerbe connection introduces a splitting in the short exact sequence

$$
0 \rightarrow T^{*} M \rightarrow E \rightarrow T M \rightarrow 0
$$

which describes the extension of $T M$ by $T^{*} M$. This allows $E$ to be split as $E=T M \oplus T^{*} M$ and to thereby identify the sections of $T M$ and $T^{*} M$ in $E$ which now transform as vectors and forms. The calculation of how this can be done has been described explicitly in [4] and amounts to going from $W$ generalised tensors to $\hat{W}$ ones in the notation of [4]. This is related to the notion of the $B$-transform in generalised geometry [20-22]. As the dual coordinates of the doubled space $\tilde{x}$ do not transform, or just transform as 1-forms, they are inert under $B$-field gauge transformations. It has been argued in [4] that to describe DFT it is sufficient to consider the diffeomorphisms of the spacetime together with the generalised geometry structure described above which includes a splitting of the exact sequence that determines the $B$ field.

\section{Patching}

Let us now turn to investigate the implications of patching doubled spaces with the transformations proposed in the previous section on the topology and geometry of spacetime. Before we do this, let us describe a few properties of the de Rham-Čech theory as applied to closed 3-forms $H$. Let $\left\{U_{\alpha}\right\}_{\alpha \in I}$ a good cover, then on the open sets $U_{\alpha}$ and the n-fold overlaps $U_{\alpha_{0} \ldots \alpha_{n-1}}=U_{\alpha_{0}} \cap \cdots \cap U_{\alpha_{n-1}}, n=2,3,4$, one has

$$
\begin{aligned}
& H_{\alpha}=d B_{\alpha}, \quad-B_{\alpha}+B_{\beta}=d a_{\alpha \beta}^{1}, \quad a_{\beta \gamma}^{1}-a_{\alpha \gamma}^{1}+a_{\alpha \beta}^{1}=d a_{\alpha \beta \gamma}^{0}, \\
& a_{\beta \gamma \delta}^{0}-a_{\alpha \gamma \delta}^{0}+a_{\alpha \beta \delta}^{0}-a_{\alpha \beta \gamma}^{0}=n_{\alpha \beta \gamma \delta},
\end{aligned}
$$

respectively, where $n_{\alpha \beta \gamma \delta}$ are constants. The last condition arises from the requirement that on 4 -fold overlaps

$$
d\left(a_{\beta \gamma \delta}^{0}-a_{\alpha \gamma \delta}^{0}+a_{\alpha \beta \delta}^{0}-a_{\alpha \beta \gamma}^{0}\right)=0 .
$$

If $n_{\alpha \beta \gamma \delta} \in 2 \pi \mathbb{Z}$, then $H$ represents a class in $H^{3}(M, \mathbb{Z})$. The left-hand sides of all but the first of equations (4.1) involve the Cech differential $\delta$. It acts on form-valued fields defined on $p$-fold overlaps and takes them to forms on $(p+1)$-fold overlaps, e.g. $(\delta B)_{\alpha \beta}=$ $-B_{\alpha}+B_{\beta} ;(\delta a)_{\alpha \beta \gamma}=a_{\alpha \beta}+a_{\beta \gamma}+a_{\gamma \alpha}$, and so on, and squares to zero, $\delta^{2}=0$.

We emphasise that the 2 -form gauge potential $B$ as well as the transition functions $a^{1}, a^{0}$ are not unique in the above decomposition. In fact the decomposition is invariant 
under the local "gauge" transformations

$$
B_{\alpha} \rightarrow B_{\alpha}+d u_{\alpha}^{1}, \quad a_{\alpha \beta}^{1} \rightarrow a_{\alpha \beta}^{1}-u_{\alpha}^{1}+u_{\beta}^{1}+d f_{\alpha \beta}^{0}, \quad a_{\alpha \beta \gamma}^{0} \rightarrow a_{\alpha \beta \gamma}^{0}+f_{\beta \gamma}^{0}-f_{\alpha \gamma}^{0}+f_{\alpha \beta}^{0},
$$

where $u^{1}$ are 1 -forms and $f^{0}$ are functions defined on the indicated overlaps.

\section{1 $B$-dependent patching for dual coordinates}

If the coordinates for the doubled space, $\tilde{x}$, are taken to be one-forms patched together using the $B$-field transformations, i.e.

$$
-\tilde{x}_{\alpha}+\tilde{x}_{\beta} \propto a_{\alpha \beta}^{1}
$$

as in $[1,2]$ and [16] (where the notation $\zeta_{\alpha \beta}$ was used for $a_{\alpha \beta}^{1}$ ), then, as shown in [12], this implies that the $H$-flux is trivial. It follows from (4.4) that $\left(\delta a^{1}\right)_{\alpha \beta \gamma}=0$ which can be solved by $a_{\alpha \beta}^{1}=\left(\delta u^{1}\right)_{\alpha \beta}$ by the $\delta$-Poincaré lemma. This in turn implies that $B_{\alpha}$ can be shifted by $\left(d u^{1}\right)_{\alpha}$ on each patch so that the new $B$-field will be globally defined. So this construction of doubled spaces is not compatible with backgrounds with non-trivial $H$-flux in $H^{3}(M, \mathbb{Z})$. There are many examples of such backgrounds, for example those discussed in section 2 .

Another patching proposal is that of [3] where it is asserted that the polarisation $b$, with $d b=0$, is defined on each patch $U_{\alpha}$ of a good cover $\left\{U_{\alpha}\right\}_{\alpha \in I}$ and patches as ${ }^{4}$

$$
b_{\alpha}=b_{\beta}+d a_{\alpha \beta}^{1},
$$

As $B$ transforms in the same way, the difference $\mathbf{B}=B-b$ transforms tensorially and one has $H=d B=d \mathbf{B}$, as $d b=0$, and so $H$ is exact.

One can reach the same conclusion by viewing the (3.5) as a patching condition on a good cover as

$$
\left(B_{\alpha}\right)_{i j}=\frac{\partial x_{\beta}^{k}}{\partial x_{\alpha}^{i}} \frac{\partial x_{\beta}^{l}}{\partial x_{\alpha}^{j}}\left(B_{\beta}-b_{\beta}\right)_{k l}+\left(b_{\beta}\right)_{i j}+\left(d v_{\alpha \beta}\right)_{i j}
$$

Since $b$ is closed, one can solve this locally as $b_{\alpha}=d u_{\alpha}$ and re-arrange the above equation using (4.5) as

$$
\left(B_{\alpha}-d u_{\alpha}\right)_{i j}=\frac{\partial x_{\beta}^{k}}{\partial x_{\alpha}^{i}} \frac{\partial x_{\beta}^{l}}{\partial x_{\alpha}^{j}}\left(B_{\beta}-d u_{\beta}\right)_{k l} .
$$

However, as we have already mentioned the definition of $B$ is ambiguous up to a gauge transformation generated by $u$. As a result $B$ can be chosen to be a globally defined 2 -form leading to an exact $H$. This result is independent from the way that the dual coordinates transform and so it is not affected by the gauge transformation introduced in [19].

An alternative reading of the proposal made in [3], which is more tuned to the examples described later in that paper, is as follows. One introduces two different 2-form gauge potentials $B$ and $\mathbf{B}$ for the 3 -form field strength $H$, but where now $\mathbf{B}$ is no longer necessarily tensorial. If the transition functions with respect to $B$ and $\mathbf{B}$ are denoted by $a^{1}$ and $a^{0}$, and $\mathbf{a}^{1}$ and $\mathbf{a}^{0}$ in the Cech-de Rham decomposition, respectively, we take the patching

\footnotetext{
${ }^{4}$ The notation $v_{\alpha \beta}$ was used for $a_{\alpha \beta}^{1}$ in [3].
} 
conditions of the dual coordinates $\tilde{x}$ to be those of the polarisation $b=B-\mathbf{B}$. These are given by $a_{\alpha \beta}^{1}-\mathbf{a}_{\alpha \beta}^{1}:=\hat{a}_{\alpha \beta}^{1}$. So one can set

$$
-\tilde{x}_{\alpha}+\tilde{x}_{\beta}=\hat{a}_{\alpha \beta}^{1}
$$

on each $U_{\alpha \beta}$. This is similar to (4.4) and implies that $\hat{a}_{\alpha \beta}^{1}=\left(\delta u^{1}\right)_{\alpha \beta}$. So if we redefine $b$ by $b_{\alpha} \rightarrow b_{\alpha}-u_{\alpha}^{1}$ on each patch $b$ will be globally defined, while if we also redefine the new coordinates in a similar fashion, $\tilde{x}_{\alpha} \rightarrow \tilde{x}_{\alpha}^{\prime}=\tilde{x}_{a}-u_{\alpha}^{1}$ the new coordinates will be inert under $b$ (or $B$ )-field gauge transformations. This is similar to the first case discussed above, but now does not require that the flux of $H$ be trivial. So this interpretation leads to a patching condition which is equivalent to one which is independent of the $B$-field patching.

\section{2 $B$-independent patching for dual coordinates}

Such a proposal is that described in [4]. The patching conditions are just the diffeomorphisms of the spacetime and the patching conditions of the generalised geometry bundle $E$ together with a choice of a splitting. The main point is that the patching conditions of the dual coordinates are

$$
\tilde{x}_{\alpha}=\tilde{x}_{\beta}
$$

i.e. they remain inert. As the generalised geometry data are by construction globally defined, the patching of such a doubled space is consistent.

However, this proposal and in particular the assertion that $\tilde{x}_{\alpha}=\tilde{x}_{\beta}$ is in conflict with the patching results that are a consequences of the Buscher T-duality rules. As we have demonstrated with an explicit calculation in section 2, a T-dual circle can topologically twist over the spacetime. As this cannot happen to the DFT dual coordinates, one can only conclude that according to this proposal DFT either does not incorporate the Buscher Tduality rules or the DFT dual coordinates $\tilde{x}$ should not be identified with the Buscher dual angular coordinates $\tilde{\theta}$. If the former is not considered desirable, then one must conclude that the DFT dual coordinates $\tilde{x}$ is not the full story and additional coordinates must be introduced. There has been such a suggestion before in [13] where the basis of generalised $\hat{W}$ generalised tensors has been identified and where it was shown how the generalised geometry emerges. In this case, one might argue that the motivation for the introduction of the DFT dual coordinates in the first place is somewhat weakened, or that they have only an auxiliary status.

To enforce the idea that a generalised geometry approach is not sufficient to describe the T-duality rules, observe that, although the generalised geometry bundle $E$ is twisted over the spacetime, as a space it is contractible to the spacetime $M$. In other words the spacetime is fixed and the bundle transformations, which one might wish to identify with T-duality transformations, cannot change the topology of the underlying space. On the other hand, we have seen that T-duality changes the topology of spacetime, for example the sphere and the lens space have different cohomology groups, and moreover both spaces in the dual pair are smooth. This does not mean that the T-duality transformation is necessarily smooth, but a smooth transformation of $E$ can never induce the T-dual geometry on the spacetime, 
i.e. only singular gauge transformations of $E$ may be of interest as they may produce the desirable T-dual space.

The modified proposal of [3] discussed above also suffers a similar problem in that the patching condition (4.8) does not reproduce the Buscher rules and cannot accommodate dual angular coordinates.

\section{A new proposal}

\subsection{C-spaces and DFT coordinates}

Here we shall propose a scenario which illustrates the role of the various coordinates and how the Buscher T-dual spaces can be incorporated using the C-space construction of [13]. Given a good cover $\left\{U_{\alpha}\right\}$ of the spacetime $M$, one introduces new coordinates $y_{\alpha}^{1}$ on every open set $U_{\alpha}$ and angular coordinates $\theta_{\alpha \beta}$ on every intersection $U_{\alpha \beta}$ and imposes the patching conditions

$$
\begin{aligned}
-y_{\alpha}^{1}+y_{\beta}^{1}+d \theta_{\alpha \beta} & =a_{\alpha \beta}^{1} \\
\left(\theta_{\alpha \beta}+\theta_{\beta \gamma}+\theta_{\gamma \alpha}+a_{\alpha \beta \gamma}^{0}\right) & =0 \bmod 2 \pi \mathbb{Z}
\end{aligned}
$$

on $U_{\alpha \beta}$ and $U_{\alpha \beta \gamma}$. Then consistency with (4.1) at triple and fourfold overlaps implies

$$
n_{\alpha \beta \gamma \delta}=0 \quad \bmod \mathbb{Z},
$$

which is satisfied provided that $\frac{1}{2 \pi} H$ represents a class in $H^{3}(M, \mathbb{Z})$. The angular coordinates at double intersections are associated with the fibre directions of the principal U(1) bundles that arise in the Hitchin-Chatterjee description of gerbes [23, 24], explained in detail in [25].

Common sector theories with $O(d, d)$ local gauge symmetry can be described solely in terms of generalised geometry, i.e. without the introduction of additional coordinates. Such theories can also be described in terms of C-spaces, as discussed in [13]. In this context of $\mathrm{C}$-spaces additional one-form coordinates can be introduced, as we have seen above, and it was shown in [13] that the first patching condition in (5.1) can be used to introduce new one-form coordinates

$$
\tilde{y}_{\alpha}^{1}=y_{\alpha}^{1}-\sum_{\gamma} \rho_{\gamma}\left(d \theta_{\alpha \gamma}-a_{\alpha \gamma}^{1}\right)
$$

which are globally defined on the spacetime, i.e. $\tilde{y}_{\alpha}^{1}=\tilde{y}_{\beta}^{1}$. Here $\left\{\rho_{\alpha}\right\}$ is a partition of unity subordinate to the good cover. It seems reasonable on the grounds of their transformation properties to identify the $\tilde{y}_{\alpha}^{1}$ with the doubled coordinates of [4] which also transform as one-forms, i.e. $\tilde{x}=\tilde{y}$, after suppressing the degree and open set labels on $\tilde{y}$. This incorporates the the DFT doubled coordinates into a C-space description.

However, we have shown that the Buscher T-dual spaces cannot be described in terms of the $(x, \tilde{x})$ coordinates alone. So the question that remains is where the Buscher T-dual spaces are hidden in this description. The C-space description contains in addition the angular coordinates $\theta$ which describe the gerbe part of the space. We shall argue that the Buscher T-dual spaces are hidden in the gerbe. 


\subsection{Gerbes and Buscher rules}

Although in the construction of C-spaces a good cover has been used, for the definition of a Hitchin-Chatterjee gerbe any open cover $^{5}$ suffices. We shall use this to adapt an open cover such that the Buscher T-duals can be described as subspaces of gerbes.

To illustrate how gerbes can be constructed, consider the example of $S^{3}$ with $N$ units of $H$ flux. We have already seen that the T-dual space of this is the lens space $L_{N}^{3}$ with one unit of flux. To describe this gerbe on $S^{3}$ [25], we can choose a stereographic cover of two open sets $\left\{U_{0}, U_{1}\right\}$ on $S^{3}$ for which their intersection $U_{0} \cap U_{1}:=U_{01}$ is $I \times S^{2}, I$ an open interval, and the Mayer-Vietoris description of $H^{3}\left(S^{3}, \mathbb{Z}\right)$ which uses representatives localised on $U_{01}$, see e.g. [26]. Such representatives are constructed as follows. As $U_{01}$ is contractible to $S^{2}$, choose a representative $F_{01}$ of the class $N u$ in $H^{2}\left(S^{2} \times I, \mathbb{Z}\right)=H^{2}\left(S^{2}, \mathbb{Z}\right)$ where $u$ is the generator of $H^{2}\left(S^{2} \times I, \mathbb{Z}\right)$. A representative of $\frac{1}{2 \pi}[H]$ can be chosen as

$$
\hat{H}_{0}=-d \rho_{1} \wedge F_{01}, \quad \hat{H}_{1}=d \rho_{0} \wedge F_{01},
$$

on $U_{0}$ and $U_{1}$, respectively, where $\left\{\rho_{0}, \rho_{1}\right\}$ is a partition of unity subordinate to the cover $\left\{U_{0}, U_{1}\right\}$. Observe that at the intersection

$$
-\hat{H}_{0}+\hat{H}_{1}=d\left(\rho_{1}+\rho_{0}\right) \wedge F_{01}=d 1 \wedge F_{01}=0,
$$

and so $\hat{H}$ is globally defined on $S^{3}$. Furthermore Stoke's theorem reveals that $[H]=[\hat{H}]$. As there are no more than double overlaps the rest of the compatibility conditions for the gerbe are trivially satisfied. The gerbe ${ }^{6}$ associated to $S^{3}$ and $H$ is then the union of $S^{3}$ together with the principal $\mathrm{U}(1)$ bundle on $U_{01}$ which has first Chern class $N u$. Observe that the principal bundle over $U_{01}$ when restricted on $S^{2} \subset U_{01} \subset S^{3}$ is the Lens space $L_{N}^{3}$. It is significant that the lens space $L_{N}^{3}$ which is the T-dual to $S^{3}$ naturally appears in this gerbe construction. Prompted by this, it is tempting to identify the T-dual angular coordinate $\tilde{\theta}$ with the fibre coordinate of the lens space that appears in the gerbe construction. We shall provide a further explanation for this below.

Suppose next that the spacetime is a product $M=S^{1} \times Q$ and the 3-form flux $H=$ $d \theta \wedge F$, where $F$ is a 2-form representing a class in $H^{2}(Q, \mathbb{Z})$ and where we have suppressed the pull back operation from $Q$ to $M$. Choose a cover on $S^{1}$ of two open sets $\left\{V_{0}, V_{1}\right\}$ then $U_{0}=V_{0} \times Q$ and $U_{1}=V_{1} \times Q$ are open and cover $M$, and their intersection $U_{01}=$ $\left(V_{0} \cap V_{1}\right) \times Q$. As $F$ is defined on $M$ it is also defined on $U_{01}$ and we denote its restriction to $U_{01}$ by $F_{01}$. Choose the gerbe principal U(1) bundle $P_{01}$ on $U_{01}$ to have Chern class represented by $F$. Then a representative of the class of the 3 -form flux $H$ on $M$ can be constructed as in equation (5.4)

$$
\hat{H}_{0}=-d \rho_{1} \wedge F_{01}, \quad \hat{H}_{1}=d \rho_{0} \wedge F_{01},
$$

where now $\left\{\rho_{0}, \rho_{1}\right\}$ is a partition of unity subordinate to the $\left\{V_{0}, V_{1}\right\}$ cover. $\hat{H}$ is globally defined on $M$ and it is a representative of the 3 -form flux associated to the gerbe. The

\footnotetext{
${ }^{5}$ Note, however, that for gerbes there is a notion of refinement [23]. As a result, any chosen open cover can be refined to a good open cover, so that any gerbe can be related to one defined on a good open cover.

${ }^{6}$ The gerbe in not a manifold. From the perspective of $S^{3}$ it grows an extra dimension as one approaches the sphere at the equator.
} 
T-dual space of $M$ is the bundle space of $P_{01}$ which is clearly a subspace of the total space of the gerbe.

As a special case of the above take $Q=T^{2}$. In this case, $M=T^{3}$ and $H$ can be chosen as in section 2.4 in which the T-dual pair of $T^{3}$ with flux was described. In particular, we set

$$
F=d p=\frac{N}{2 \pi} d \psi_{2} \wedge d \psi_{3} .
$$

In this case, the restriction of the principal $\mathrm{U}(1)$ gerbe bundle $P_{01}$ on $Q=T^{2} \subset U_{01} \subset T^{3}$ is the T-dual space $\tilde{T}^{3}$ as described by the Buscher T-duality rules. For a different treatment of this example, see [5].

As a final example we take $M$ to be a circle bundle over $Q$ with 3 -form flux $H$ that can be represented as $[H]=a w$, where $w \in H^{2}(Q, \mathbb{Z})$ and $a$ is the generator of $H^{1}\left(S^{1}, \mathbb{Z}\right)$. Take an open cover $\left\{W_{\alpha}\right\}$ on $Q$ which trivialises the circle bundle $M$ over $Q$, i.e. $\pi^{-1}\left(W_{\alpha}\right)=$ $\varphi_{\alpha}^{-1}\left(W_{\alpha} \times S^{1}\right)$, where $\pi: M \rightarrow Q$ is the projection and $\varphi_{\alpha}: \pi^{-1}\left(W_{\alpha}\right) \rightarrow W_{\alpha} \times S^{1}$ is the trivilisation map, and write each $W_{\alpha} \times S^{1}$ as the union of the open set $W_{\alpha} \times V_{0}$ and $W_{\alpha} \times V_{1}$, where $\left\{V_{0}, V_{1}\right\}$ are the two open sets that cover $S^{1}$ introduced above. It is clear that $\left\{\varphi_{\alpha}^{-1}\left(W_{\alpha} \times V_{r}\right)\right\}, r=0,1$, is a cover for $M$. As the union of open sets is open $U_{0}=$ $\bigcup_{\alpha} \varphi_{\alpha}^{-1}\left(W_{\alpha} \times V_{0}\right)$ and $U_{1}=\bigcup_{\alpha} \varphi_{\alpha}^{-1}\left(W_{\alpha} \times V_{1}\right)$ are open and cover $M$. As in the case that $M$ was a product, we consider a representative $F$ of the class $w \in H^{2}(Q)$ and its pull back to $M$ with the projection map $\pi$. Restricting $F$ to the intersection of $U_{01}$ and denoting it by $F_{01}$, we can construct a representative $\hat{H}$ of the $H$ flux as in (5.4), where again $\left\{\rho_{0}, \rho_{1}\right\}$ is a partition of unity subordinate to the cover $\left\{U_{0}, U_{1}\right\} . \hat{H}$ is globally defined and represents $[H]=a w$ as the derivatives of the partition functions at the intersection of open sets on the circle represent the generator of $H^{1}\left(S^{1}, \mathbb{Z}\right)$. It is clear that the gerbe is the union of spacetime with a circle bundle defined on the open set $U_{01}$ of $M$ which is the restriction of the pull-back of a circle bundle over the base space $Q$ with Chern class $w$. The circle bundle over $Q$ is the T-dual space derived from the Buscher rules. If $S^{3}$ is viewed as a circle fibration over $S^{2}$ and $H$ represents $N$ units of flux, the above gerbe construction will also lead to the identification of the T-dual space as $L_{N}^{3}$. It is clear that the gerbes in all the above examples have simple descriptions because the spacetimes have been covered by only two open sets.

\subsection{Summary of the proposal}

The above results provide evidence to suggest that the double coordinates $\tilde{x}$ of DFT that transform like 1-forms [4] should be identified with the $\tilde{y}$ coordinates that occur in C-spaces, eqn (5.3). DFT can be formulated with only these coordinates and will exhibit local $O(d, d)$ symmetry as such a description accommodates generalised geometry both from the double spaces point of view and that of C-spaces. However, such a formulation will not describe the T-dual spaces of the spacetime. This is regardless of the choice of solution to the strong section condition that one makes on the doubled space.

Our results have also established that the T-dual space of a spacetime with $H$ flux can be identified as a subspace of a gerbe which is part of the C-space. This has been done explicitly for the T-dual space derived after performing T-duality along the fibre direction of a spacetime which is a circle fibre bundle. This identification requires the presence of 
additional coordinates from those of doubled space which are the fibre coordinates of the principal $\mathrm{U}(1)$ bundles that lie on double intersections of an open cover of the spacetime and are required in the description of the gerbe.

We have given two gerbe descriptions of the T-dual space of $S^{3}$ with $N$ units of $H$ flux example. The first description was in terms of a stereographic cover and the other in terms of a cover adapted to the fibration over $S^{2}$. In both cases, the T-dual space has been identified as the lens space $L_{N}^{3}$. In hindsight this may have been expected. The T-dual space should be independent from a large enough selection of covers on the spacetime that are used to describe the gerbe. This can be seen as the requirement for the construction of gerbes and that of T-dual spaces to be covariant. In turn one can view this as a covariant description of the Buscher T-duality rules.

\section{Conclusions}

We have made a proposal based on C-spaces and the Hitchin-Chatterjee description of a gerbe where both the local (bundle) $O(d, d)$ symmetry and the T-dual spaces of a spacetime can be described in a globally consistent way. In particular, we have demonstrated that the doubled space of a DFT as described in [4] can be included into a C-space and the $O(d, d)$ symmetry arises as part of the generalised geometry structure on C-spaces. Furthermore, we have demonstrated that the T-dual spaces of a spacetime that are constructed using Buscher rules can be identified as subspaces of the gerbe which is included in C-spaces but not in the doubled spaces. In this identification, the T-dual angular coordinate of a spacetime which is a circle fibration with T-duality operation taken along the fibre circle is identified with the gerbe angular coordinate which is the fibre coordinate of a principal $\mathrm{U}(1)$ bundle defined on an intersection of two open sets of the spacetime.

Our analysis has indicated that it is not possible to formulate a theory which exhibits both local $O(d, d)$ symmetry and at the same time has a description of all the T-dual spaces of a spacetime based only on doubled spaces. Using the available globally consistent definitions of doubled spaces, we have demonstrated that these cannot provide an explanation for the property of the T-dual circles to topologically twist over the spacetime. This topological twisting has been established in several examples and it is a consequence of the Buscher rules. In other words, the T-dual spaces cannot arise in DFT as different solutions to the (strong) section condition on doubled spaces.

The inclusion of gerbes in a consistent definition of a theory which exhibits local $O(d, d)$ symmetry and which describes the T-dual spaces of a spacetime requires the presence of additional angular coordinates, the gerbe coordinates. Such spaces are not manifolds and in particular they do not have a fixed dimension. Nevertheless they contain all the necessary ingredients for the definition of the theory including the ability to perform differential geometry computations related to $O(d, d)$ symmetry and the topological properties required for the descrption of the T-dual spaces.

The gerbe description of T-dual spaces of a spacetime has some additional consequences. First notice that the Buscher rules are not covariant. Their formulation involves several gauge choices and their construction is essentially local on the spacetime. Moreover, 
they depend on the spacetime admitting an isometry. On the other hand gerbes can be defined on any smooth manifold with a closed 3-form flux $H$ without further additional assumptions. Therefore the gerbe description can be seen as a covariantisation of the Tduality rules. Furthermore the gerbe description opens the possibility that it might be possible to investigate the T-duals of a spacetime that does not admit isometries. In this case, however, it may not be possible to identify the subspaces of the gerbe which can be characterised as T-dual spaces as we have done in the case of spacetimes with isometries. Even if the T-dual spaces can be identified, it is likely that they will not be manifolds.

\section{Acknowledgments}

GP is partially supported by the STFC rolling grant ST/J002798/1.

Open Access. This article is distributed under the terms of the Creative Commons Attribution License (CC-BY 4.0), which permits any use, distribution and reproduction in any medium, provided the original author(s) and source are credited.

\section{References}

[1] O. Hohm and B. Zwiebach, Large gauge transformations in double field theory, JHEP 02 (2013) 075 [arXiv: 1207.4198] [INSPIRE].

[2] O. Hohm, D. Lüst and B. Zwiebach, The spacetime of double field theory: review, remarks and outlook, Fortsch. Phys. 61 (2013) 926 [arXiv:1309.2977] [INSPIRE].

[3] S.-J. Rey and Y. Sakatani, Finite transformations in doubled and exceptional space, arXiv: 1510.06735 [INSPIRE].

[4] C.M. Hull, Finite gauge transformations and geometry in double field theory, JHEP 04 (2015) 109 [arXiv: 1406.7794] [INSPIRE].

[5] F. Hassler, The topology of double field theory, arXiv:1611.07978 [INSPIRE].

[6] R. Blumenhagen, F. Hassler and D. Lüst, Double field theory on group manifolds, JHEP 02 (2015) 001 [arXiv: 1410.6374] [INSPIRE].

[7] R. Blumenhagen, P. du Bosque, F. Hassler and D. Lüst, Generalized metric formulation of double field theory on group manifolds, JHEP 08 (2015) 056 [arXiv: 1502.02428] [INSPIRE].

[8] M.J. Duff, Duality rotations in string theory, Nucl. Phys. B 335 (1990) 610 [INSPIRE].

[9] A.A. Tseytlin, Duality symmetric closed string theory and interacting chiral scalars, Nucl. Phys. B 350 (1991) 395 [inSPIRE].

[10] W. Siegel, Two vierbein formalism for string inspired axionic gravity, Phys. Rev. D 47 (1993) 5453 [hep-th/9302036] [INSPIRE].

[11] W. Siegel, Superspace duality in low-energy superstrings, Phys. Rev. D 48 (1993) 2826 [hep-th/9305073] [INSPIRE].

[12] G. Papadopoulos, Seeking the balance: patching double and exceptional field theories, JHEP 10 (2014) 089 [arXiv: 1402.2586] [INSPIRE].

[13] G. Papadopoulos, C-spaces, generalized geometry and double field theory, JHEP 09 (2015) 029 [arXiv: 1412.1146] [INSPIRE]. 
[14] M. Cederwall, T-duality and non-geometric solutions from double geometry, Fortsch. Phys. 62 (2014) 942 [arXiv: 1409.4463] [INSPIRE].

[15] M. Cederwall, The geometry behind double geometry, JHEP 09 (2014) 070 [arXiv: 1402.2513] [INSPIRE].

[16] D.S. Berman, M. Cederwall and M.J. Perry, Global aspects of double geometry, JHEP 09 (2014) 066 [arXiv:1401.1311] [INSPIRE].

[17] D.M. Belov, C.M. Hull and R. Minasian, T-duality, gerbes and loop spaces, arXiv:0710.5151 [INSPIRE].

[18] P. Bouwknegt, J. Evslin and V. Mathai, On the topology and $H$ flux of $T$ dual manifolds, Phys. Rev. Lett. 92 (2004) 181601 [hep-th/0312052] [INSPIRE].

[19] J.-H. Park, Comments on double field theory and diffeomorphisms, JHEP 06 (2013) 098 [arXiv: 1304.5946] [INSPIRE].

[20] N. Hitchin, Generalized Calabi-Yau manifolds, Quart. J. Math. 54 (2003) 281 [math/0209099] [INSPIRE].

[21] N. Hitchin, Brackets, forms and invariant functionals, math/0508618 [INSPIRE].

[22] M. Gualtieri, Generalized complex geometry, Ph.D. thesis, Oxford University, Oxford U.K., (2003) [math/0401221] [INSPIRE].

[23] D.S. Chatterjee, On gerbs, dissertation submitted towards the degree of Doctor of Philosophy, University of Cambridge, Trinity College, Cambridge U.K., (1998).

[24] N.J. Hitchin, Lectures on special Lagrangian submanifolds, math/9907034 [INSPIRE].

[25] M.K. Murray, An introduction to bundle gerbes, arXiv:0712.1651 [INSPIRE].

[26] R. Bott and L.W. Tu, Differential forms in algebraic topology, page 36, Springer-Verlag, New York U.S.A., (1982). 